\title{
MicroRNAs: key gene regulators with versatile functions
}

\author{
Luca Comai $\cdot$ Baohong Zhang
}

Published online: 24 July 2012

(C) Springer Science+Business Media B.V. 2012

MicroRNAs (miRNAs) are a recently discovered class of small endogenous RNAs, which negatively regulate gene expression at the post-transcriptional levels. Since their discovery in 2002, plant miRNAs have been recognized as one of the most important gene regulators. Multiple evidences indicate that miRNAs play an important role in almost all plant cellular and metabolic processes, including developmental timing, signal transduction, cell maintenance and differentiation as well as response to biotic and abiotic stresses. Therefore, microRNA-related research has become one of the hottest areas in plant biology.

This special issue is dedicated to the rapidly expanding research field on small RNAs, particularly on miRNAs and it features five review papers, three research articles and also one software paper. The review paper focuses on the different aspects of miRNA conservation, divergence, and functions as well as the status of art in moss, wheat and tree microRNA. The research articles address miRNA functions in male gametophyte development, the interaction between miRNAs and trans-acting RNAs, and the potential of premiRNA 159a to encode a second mature miRNAs with differentially expressed pattern. The software paper presents a comprehensive software packages for identifying and functionally analyzing miRNAs from deep sequencing datasets.

We greatly appreciate the contribution of all authors and manuscript reviewers; they are all experts in this field. We also truly appreciate the support and help of the Editor-inChief and the Editorial Office staff of Plant Molecular Biology. Without their support, this specific issue could not have been possible.
L. Comai

Plant Biology and Genome Center, University of California, Davis, 451 Health Sciences Drive, Davis, CA 95616, USA

e-mail: 1comai@ucdavis.edu

B. Zhang $(\bowtie)$

Department of Biology, East Carolina University, Greenville, NC 27858, USA

e-mail: zhangb@ecu.edu 